\title{
Co-évolution des paysages urbain et agraire à Ouidah (Sud-ouest du Bénin) : entre urbanisation et déprise agricole
}

\section{Ahokpossi M. R. Ghislain, Doctorant}

Département de Géographie et Aménagement du Territoire (DGAT),

Faculté des Sciences Humaines et Sociales (FASHS),

Université d'Abomey-Calavi (UAC, Bénin)

Yolou Isidore, Maître-Assistant

Département de Géographie et Aménagement du Territoire (DGAT),

Faculté des Lettres, Arts et Sciences Humaines (FLASH),

Université de Parakou (UP, Bénin)

Ouorou Yerima Guèguè Léa, Doctorante

Yabi Ibouraima, Maître de Conférences

Département de Géographie et Aménagement du Territoire (DGAT),

Faculté des Sciences Humaines et Sociales (FASHS),

Université d'Abomey-Calavi (UAC, Bénin)

Akiyo Offin Lié Rufin, Maître de Conférences

Département de Sociologie et Anthropologie (DSA), Faculté des Lettres, Arts et Sciences Humaines (FLASH), Université de Parakou (UP, Bénin)

Yabi Afouda Jacob, Professeur Titulaire

Laboratoire d'Analyses et de Recherches sur les Dynamiques Economiques

et Sociales (LARDES), Faculté d'Agronomie (FA),

Université de Parakou (UP), Bénin

Afouda Fulgence, Professeur Titulaire

Département de Géographie et Aménagement du Territoire (DGAT),

Faculté des Sciences Humaines et Sociales (FASHS),

Université d'Abomey-Calavi (UAC, Bénin)

Doi:10.19044/esj.2020.v16n14p143 URL:http://dx.doi.org/10.19044/esj.2020.v16n14p143

\section{Résumé}

Les dynamiques foncières dues principalement à l'urbanisation ont profondément modifié l'occupation et l'organisation des espaces urbain et rural. La présente recherche vise à analyser la double évolution du paysage urbain et des espaces cultivables en vue d'une contribution à la maitrise du phénomène d'urbanisation et ses impacts sur l'agriculture. Les données utilisées sont relatives à l'évolution démographique de la Commune de Ouidah 
et son influence sur les terres agricoles et aux unités d'occupation du sol de 1995 et de 2010. En plus de la recherche documentaire, les investigations de terrain ont été faites. A cet effet, un effectif de 212 chefs d'exploitation agricole répartis dans cinq (5) arrondissements, a été pris en compte suivant un choix raisonné. Le calcul de l'Indice d'Evolution (IE) et quelques paramètres de la statistique descriptive combinés avec l'approche cartographique ont permis de traiter les données collectées. Il ressort des résultats que la location (49\%) reste le mode d'accès à la terre le plus dominant suivi de l'héritage (38,2\%). Les modes d'accès tels que le don $(2,80$ $\%)$, l'achat $(9,90 \%)$ sont d'une proportion très faible. Entre 1995 et 2010, l'agglomération est passée de $6,1 \mathrm{~km}^{2}$ à $15,14 \mathrm{~km}^{2}$ soit une augmentation de $148,19 \%$ au détriment de la plantation, des champs et jachères sous palmeraie, des champs et jachères, de la formation marécageuse et de la plage sablonneuse. Le mouvement d'urbanisation dans la Commune de Ouidah prend alors de l'ampleur au fil des années et les superficies cultivables s'amenuisent de plus en plus. Face à cette accélération de l'urbanisation des espaces ruraux périphériques, il s'avère indispensable que les pouvoirs publics adoptent des politiques de planification spatiale en vue d'une cohabitation équilibrée des paysages urbain et agraire dans la Commune de Ouidah.

Mots clés : Ouidah (Bénin), urbanisation, paysage urbain, paysage agraire, déprise agricole 


\title{
Co-evolution of urban and agrarian landscapes in Ouidah (South-west of Benin): between urbanization and agricultural decline
}

\author{
Ahokpossi M. R. Ghislain, Doctorant \\ Département de Géographie et Aménagement du Territoire (DGAT), \\ Faculté des Sciences Humaines et Sociales (FASHS), \\ Université d'Abomey-Calavi (UAC, Bénin) \\ Yolou Isidore, Maître-Assistant \\ Département de Géographie et Aménagement du Territoire (DGAT), \\ Faculté des Lettres, Arts et Sciences Humaines (FLASH), \\ Université de Parakou (UP, Bénin) \\ Ouorou Yerima Guèguè Léa, Doctorante \\ Yabi Ibouraima, Maître de Conférences \\ Département de Géographie et Aménagement du Territoire (DGAT), \\ Faculté des Sciences Humaines et Sociales (FASHS), \\ Université d'Abomey-Calavi (UAC, Bénin) \\ Akiyo Offin Lié Rufin, Maître de Conférences
}

Département de Sociologie et Anthropologie (DSA), Faculté des Lettres, Arts et Sciences Humaines (FLASH), Université de Parakou (UP, Bénin)

Yabi Afouda Jacob, Professeur Titulaire

Laboratoire d'Analyses et de Recherches sur les Dynamiques Economiques

et Sociales (LARDES), Faculté d'Agronomie (FA),

Université de Parakou (UP), Bénin

Afouda Fulgence, Professeur Titulaire

Département de Géographie et Aménagement du Territoire (DGAT),

Faculté des Sciences Humaines et Sociales (FASHS),

Université d'Abomey-Calavi (UAC, Bénin)

Abstract
Land tenure dynamics mainly caused by urbanization have deeply
modified the occupation and organization of urban and rural areas. The present
study aims to analyze the development of both the urban landscape and the
cultivated areas, in order to help control the urbanization phenomenon and its
impacts on agriculture. The data used are based on the demographic growth of
Ouidah Township, and its influence on the cultivated areas as well as soil
occupation, in 1995 and 2010 . In addition to the review of literatures, field 
investigation was also carried out. To achieve this, 212 farmers from 5 districts were interviewed. Growth index (GI), and some descriptive statistical parameters, combined with mapping approach were used to analyze the collected data. The obtained results show that the lease of lands (49\%) was the most dominant mode of land acquisition, followed by inheritance (38.2 $\%)$. The other modes of land acquisition were donation (2.80 \%) and purchase (9.90\%). Between 1995 and 2010, the urban area ranged from $6.1 \mathrm{~km}^{2}$ to $15.14 \mathrm{~km}^{2}$, which represents $148.19 \%$ increase, to the detriment of plantations, farms, palm trees, fallows, swamp and sandy beaches. The urbanization growth in Ouidah Township has gained momentum over the years, while cultivated areas have decreased. With such high speed in urbanization of the peripheral rural areas, it is crucial that official authorities plan the management of the lands, for proper balanced cohabitation of urban and agrarian lands in the Township of Ouidah.

Keywords: Ouidah (Benin), urbanization, urban landscape, agrarian landcapes, agricultural decline

\section{Introduction}

L'Afrique de l'Ouest est l'une des dernières régions du monde à ne pas avoir achevé sa transition démographique (T. Allen et al., 2015, p. 3). Entre 1950 et 2015, sa population passe de 70 millions à 350 millions d'habitants. Elle représente $29 \%$ de la population du continent africain et $45 \%$ de la population ouest-africaine qui vit en ville (T. Allen et al., 2015, p. 6). La population urbaine des pays d'Afrique subsaharienne est estimée à 300 millions en 2020 avec un taux de croissance de 3,7 \% par an (J. Veron, 2007 cité par M. Kanda et al., 2017, p.1). Depuis les années 60, cette partie de l'Afrique connaît le taux le plus élevé d'urbanisation au monde et cela, malgré le fait que cette région soit majoritairement rurale (J. L. Piermay, 2003 cité par A. G. Glèlè, 2015, p. 13). La croissance urbaine de l'Afrique de l'Ouest ne concerne pas que les grandes métropoles mais aussi les centres urbains de petite et moyenne tailles (T. Allen et al., 2015, p. 7). En conséquence, la distance moyenne qui sépare les agglomérations urbaines passe de $111 \mathrm{~km}$ à $33 \mathrm{~km}$ entre 1950 et 2010. Cette croissance urbaine se traduit par une forte demande en terres à bâtir et est largement alimentée par le développement des habitations individuelles (A. Guézéré, 2011, cité par M. Kanda et al., 2017, p. 2). Ainsi, l'étalement des villes engendre une consommation foncière au détriment des espaces agricoles.

Selon le PNUD (2015, p. 5), le secteur agricole au Bénin est dominé par de petites et moyennes exploitations agricoles, dont le nombre est estimé à environ 550000 exploitants agricoles qui assurent, à eux seuls, $95 \%$ de la production agricole. Avec l'accroissement démographique associée à 
l'urbanisation, les espaces agricoles disponibles par exploitant se réduisent de génération en génération (A. Y. Tohozin et al., 2012, p. 5 ; J. Ruiz et G. Domon, 2005, p. 5). Dans ce contexte, de nouvelles agglomérations naissent de la coalescence de villages et de la densification de l'habitat en milieu rural. Dans les localités rurales plus ou moins proches des grandes agglomérations, la terre est sujette à une demande accrue et fait l'objet d'intenses transactions à travers des systèmes d'achat et de vente et des processus d'accaparement individualisé (FAO, 2007 cité par A. Y. Tohozin et $a l ., 2012$, p. 2). Les risques éventuels d'une précarité de l'agriculture, dans le contexte d'urbanisation au Bénin sont énormes eu égard à l'évolution du taux d'urbanisation qui est de $26,5 \%$ en $1979 ; 36 \%$ en 1992 et $38,85 \%$ en 2002 (H. Kakaï, 2009 cité par A. Y. Tohozin et al., 2012, p. 2) et 44,6\% en 2015 (INSAE, 2018, p. 13).

Face à une population agricole qui ne cesse de perdre ses terres, une dissociation croissante s'opère ainsi entre la dynamique des territoires, réalité visible du paysage, et celle des usages et valeurs des différents segments de la population (J. Ruiz et G. Domon, 2005, p. 5). Ainsi, l'occupation de l'espace rural continue de se modifier (T. Allen et al., 2015, p. 9). Une partie des zones agricoles ou naturelles des périphéries urbaines se transforment désormais en zones dites « artificielles », principalement sous l'effet de la construction de logements individuels (B. Baccaïni et F. Semecurbe, 2009, p.1). Cette situation est due au fait que les relations entre la gestion de la terre et la dynamique des espaces agricoles sont étroites au vu des mouvements qui les animent (A. Y. Tohozin et $a l ., 2012$, p. 7). La pression foncière se traduit ainsi par l'émiettement des espaces agricoles et l'acquisition des terres par les citadins. En effet, les villes béninoises se développent rapidement, grignotent la ceinture verte périphérique ; ce qui engendre des conflits d'usage de la terre (A. Y. Tohozin et $a l ., 2012$, p. 7). La rareté ou la pénurie des terres autour de la ville de Ouidah oblige spécifiquement certains habitants à s'installer dans les localités environnantes où des paysans ont immigré à la recherche de terres fertiles (op. cit). Le présent article analyse les nouvelles dynamiques paysagiques qui naissent de l'expansion de la ville en vue d'envisager une coévolution qui assure un développement local durable de la Commune de Ouidah.

\subsection{Présentation de l'espace d'étude}

La Commune de Ouidah est située au sud du département de $l^{\prime}$ Atlantique entre $2^{\circ}$ et $2^{\circ} 15$ de longitude Est et $6^{\circ} 15$ et $6^{\circ} 30$ de latitude Nord. D'une superficie de $364 \mathrm{~km}^{2}$, elle est limitée au sud par l'Océan Atlantique, à l'est par la Commune d'Abomey Calavi, à l'Ouest par la Commune de GrandPopo et au Nord par les Communes de Kpomassè et Tori-Bossito (figure 1). 
Figure 1. Situation géographique et administrative de la zone d'étude

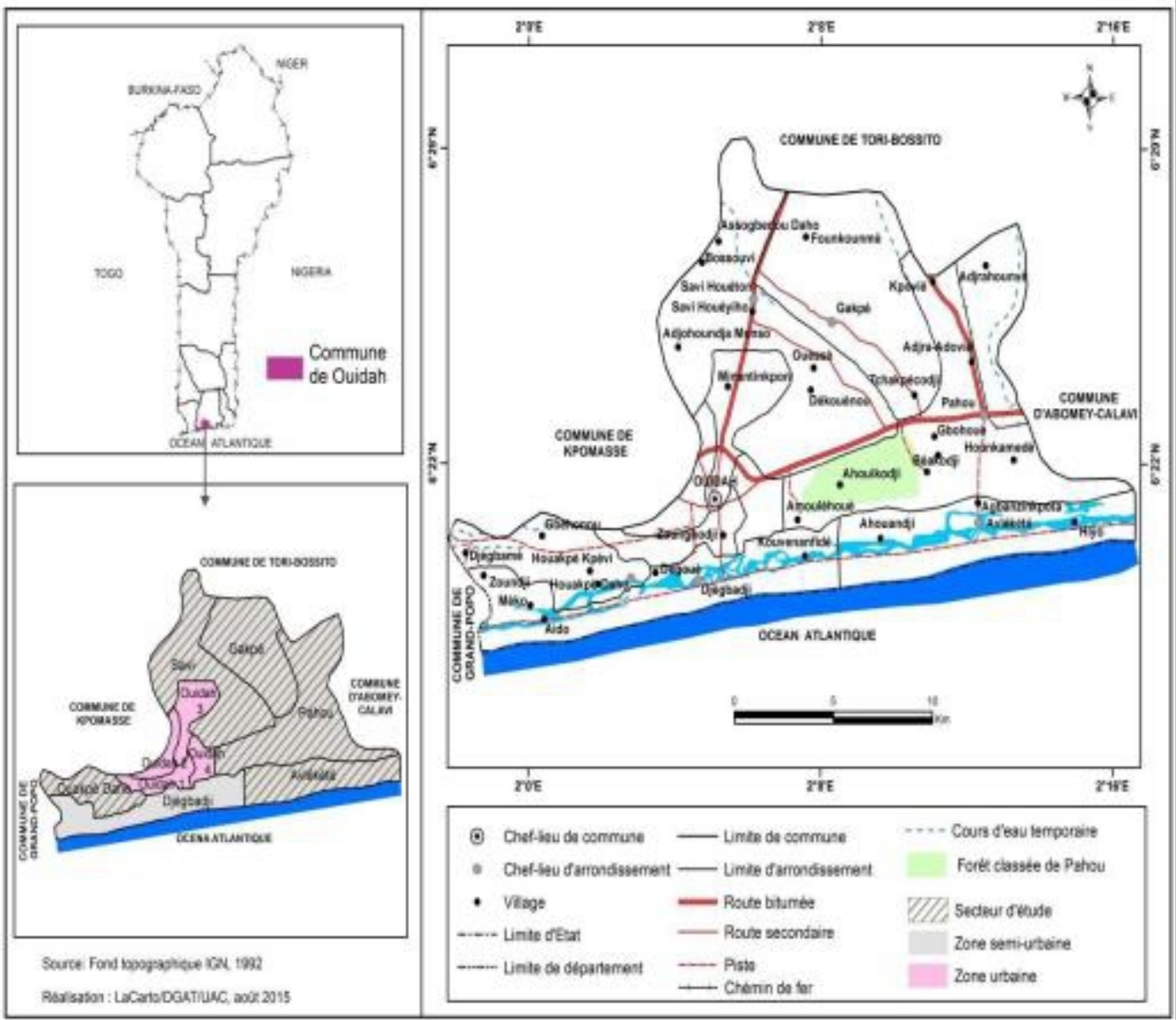

La Commune de Ouidah compte 60 quartiers de ville et villages répartis sur 10 arrondissements que sont : Ouidah I, Ouidah II, Ouidah III, Ouidah IV, Avlékété, Djègbadji, Gakpé, Houakpè-Daho, Pahou, Savi.

Le $4{ }^{\text {ème }}$ Recensement Général de la Population et de l'Habitation (RGPH) de 2013 estime la population de la Commune de Ouidah à 161544 habitants contre 76555 habitants en 2002, 64433 habitants en 1992 et 52584 habitants en 1979, soit un taux d'accroissement de de $1,46 \%$ entre 1979 et 1992 , de $1,74 \%$ entre 1992 et 2002 et de 6,86 \% entre 2002 et 2013. D'une densité de 444 habitants (hbts) $/ \mathrm{km}^{2}$, elle comprend une population agricole de 17061 habitants. Cette croissance démographique induit deux implications fondamentales : l'augmentation de la demande alimentaire et le grignotage des espaces agricoles (les terres cultivables deviennent rares) au profit des espaces bâtis.

Au niveau pédologique, la Commune est dominée par les sols sablonneux et ferralitiques. Les sols sablonneux se retrouvent non seulement au niveau du cordon littoral (Avlékété, Djègbadji et Ouakpè-Daho) mais 
également dans la partie méridionale (Ouidah I, Ouidah III et Pahou). Par contre, les sols ferralitiques sont généralement localisés à Savi, à Gakpé et la partie septentrionale des arrondissements urbains (Ouidah I, Ouidah II, Ouidah III, Ouidah IV). Entre ces deux types de sols, existe la frange lagunaire, constituée de zones marécageuses et de plaine d'inondation. De façon générale, les sols de la Commune de Ouidah présentent de bonnes aptitudes pour plusieurs types de cultures comme les tubercules, racines, céréales, légumineuses. Le relief de ce milieu est relativement plat avec un dénivelé d'environ $37 \mathrm{~m}$ entre la côte de l'océan Atlantique et le point le plus élevé. Ce relief fait partie de la plaine côtière qui est reliée au plateau d'Allada par un talus. Les pentes qui s'y trouvent sont généralement moins de $5 \%$. Les formations géologiques qui composent ce relief sont caractérisées par des dépôts de graviers alluviaux de faible épaisseur et contiennent également du sable et de l'argile sur la partie nord de la Commune. La côte est caractérisée par des dépôts littoraux anciens composés de sable et de dépôts mis en place par les marées et constitués d'argile, de sable et de niveaux charbonneux.

Le couvert végétal est essentiellement dominé par des mosaïques de cultures et des jachères à palmiers. La flore est d'une manière générale dégradée, entièrement défrichée et qui laisse apparaitre par endroits, l'existence de quelques îlots de forêts sacrées comme celles de Kpassè-Zoumè et d'Avlékété. En dehors de ces reliques de forêts naturelles, il existe de grandes plantations de palmier à huile, de cocotiers, d'arbres fruitiers (notamment les manguiers), de bois de feu. La cocoteraie reste le type de végétation du cordon littoral. Les formations naturelles sont la savane herbeuse, les prairies et les formations marécageuses à Raphia gigantea, quelques mangroves à Rhizophora racemosa et à Avicenia africana. Ces formations naturelles, notamment certaines espèces ou formations constituent les indicateurs de reconnaissance de l'état de fertilité des terres chez les exploitants agricoles (CIRADeL, 2012).

Le climat de la Commune de Ouidah est de type subéquatorial. Il est également caractérisé par deux saisons pluvieuses : la grande de mars à juillet et la petite de septembre à octobre. Au cours de ces deux périodes, les flux de mousson et les lignes de grains sont dominants. Les activités de production agricole s'y déroulent normalement. Mais, les pluies torrentielles peuvent causer des inondations. Entre les deux saisons pluvieuses, des événements de sécheresse interviennent de novembre à mars pendant la grande saison pluvieuse et en août pendant la petite saison pluvieuse. La température varie de $24^{\circ}$ à $30^{\circ} \mathrm{C}$, en saisons des pluies et de $23^{\circ}$ à $33^{\circ} \mathrm{C}$, en saisons sèches avec une moyenne annuelle de $27^{\circ} \mathrm{C}$. Ces valeurs correspondent aux besoins thermiques de toutes les plantes cultivées dans la Commune (CIRADeL, 2012). La période de croissance végétale des cultures à cycle long oscille autour de 240 jours. 
En définitive, la Commune de Ouidah, dispose d'importants atouts naturels pour l'exploitation agricole. Ce potentiel naturel qui offre une disponibilité des terres agricoles, subit l'impact de la dynamique spatiale du milieu urbain.

\section{Collecte des Données et méthodes d'analyse}

Les données utilisées concernent les variables qualitatives et quantitatives qui décrivent l'évolution démographique dans la Commune de Ouidah et son influence sur les terres agricoles. Il s'agit des statistiques démographiques issues des Recensements Généraux de la Population et de l'Habitation (RGPH) des années 1979, 1992, 2002 et 2013, collectées au centre de documentation de l'Institut National de la Statistique et de l'Analyse Economique (INSAE) et des statistiques agricoles extraites de la base de données du pôle de développement agricole $\mathrm{n}^{\circ} 7$. Les données portant sur le nouveau code foncier rural en République du Bénin et les fonds des cartes d'occupation du sol ont également été exploités. Les images LANDSAT de 1995 et de 2010 disponibles ont été utilisées pour étudier la dynamique de l'occupation du sol.

Les données relatives à la répartition spatio-temporelle de la population comme facteurs de pression sur les ressources naturelles ont été collectées. Ainsi, les entretiens individuels et de groupes (focus group), ont été réalisés à l'aide d'un questionnaire et d'un guide d'entretien. Pour mieux comprendre les dynamiques foncières impulsées par les dynamiques urbaines, les observations directes, ont été faites dans les exploitations agricoles et les milieux périurbains où la pression foncière est exercée. L'échantillon d'investigations est déterminé en considérant les ménages agricoles par choix raisonné à base de sondage suivant trois (03) critères que sont : l'expérience d'au moins trois (03) ans dans la production agricole, l'importance de la superficie emblavée et la position géographique. Les enquêtes ont été réalisées au niveau des exploitants agricoles dans 16 villages répartis dans cinq (5) arrondissements de la Commune de Ouidah (Avlékété, Gakpé, Houakpè, Pahou, et Savi) choisis sur la base de l'importance des activités agricoles dans la zone périurbaine. La taille de l'échantillon est déterminée à partir de la formule de Schwartz (1995) $: \mathrm{n}=\mathrm{Z} \alpha 2 \times \mathrm{pq} / \mathrm{i} 2$ avec $: \mathrm{n}=$ taille de l'échantillon ; $\mathrm{Z} \alpha=1,96$ est l'écart réduit correspondant à un risque $\alpha$ de $5 \% ; \mathrm{p}=\mathrm{n} / \mathrm{N} ; \mathrm{p}=$ proportion des ménages (n) des cinq arrondissements les plus réputés aux activités agricoles périurbaines par rapport au nombre de ménages dans la Commune $(\mathrm{N})$ choisi dans chaque village suivant la formule de quotas; $\mathrm{q}=$ $1-\mathrm{p}$ et $\mathrm{i}=$ précision désirée égale à $5 \%$. Au total 212 ménages agricoles ont été enquêtés sur un effectif total 1573 ménages soit 13,47 \% (tableau 1). 
Tableau 1 : Répartition des personnes enquêtées

\begin{tabular}{|c|c|c|c|}
\hline Arrondissements & Villages & $\begin{array}{l}\text { Effectif des chefs de } \\
\text { ménages agricoles }\end{array}$ & $\begin{array}{l}\text { Nombre des chefs de } \\
\text { ménages agricoles enquêtés }\end{array}$ \\
\hline \multirow[t]{3}{*}{ Avlékété } & Agbanzinkpota & 50 & 7 \\
\hline & Avlékété & 86 & 13 \\
\hline & Amouléhoué & 105 & 16 \\
\hline \multirow[t]{3}{*}{ Gakpé } & Fonkounmè & 111 & 17 \\
\hline & Gakpé & 163 & 24 \\
\hline & Tchiakpècodji & 107 & 16 \\
\hline Houakpè & Gbéhonou & 54 & 8 \\
\hline \multirow[t]{5}{*}{ Pahou } & Adjra-Adovié & 55 & 8 \\
\hline & Kpovié & 93 & 14 \\
\hline & AdjohoundjaMonso & 85 & 13 \\
\hline & Assogbénou Daho & 124 & 19 \\
\hline & Bossouvi & 101 & 15 \\
\hline \multirow[t]{4}{*}{ Savi } & Dékouénou & 81 & 12 \\
\hline & Ouessè & 71 & 11 \\
\hline & Houéton & 31 & 5 \\
\hline & Houéyiho & 96 & 14 \\
\hline Total & & 1573 & 212 \\
\hline
\end{tabular}

Source : Résultats des travaux de terrain, 2018

Les données quantitatives collectées sur le terrain ont été codifiées et saisies dans le tableur Excel 2007 puis transférées dans le logiciel SPSS version 17.0 pour leur traitement. Ainsi, sur la base des requêtes, la description de la structure et de la dynamique du paysage a intégré l'évaluation de l'état du paysage à partir des cartes d'occupation du sol de 1995 et de 2010 en format vecteur (figure 2).

Figure 2 : Chaîne de traitement de données dérivées pour la spatialisation

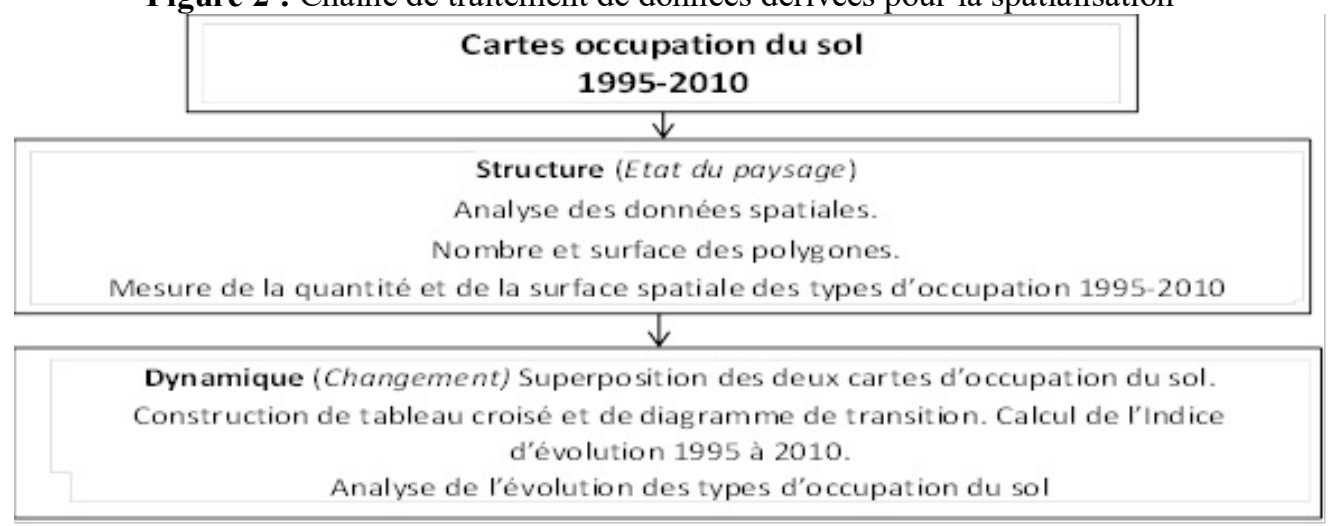

Source : Adapté de la chaîne de traitement de A. CASADO, 2007, p. 15 
Cette recherche a également pris en compte l'analyse de la distribution spatiale des polygones représentant un type d'occupation de sol donné et les mesures de surface. Pour chaque année, les Surfaces Planes (SP) des polygones ont été calculées à partir d'une Geodatabase définie a priori. Ainsi, les changements de l'état du paysage dans le temps ont été établis par la superposition des cartes en format vecteur (outil intersect) dans les années 1995 à 2010. La multiplicité de catégories produit de la superposition spatiotemporelle a été corrigée par la discrimination de transitions impossibles et/ou pas significatives, par la construction de tableaux croisés et de la matrice de transition.

Les tableaux croisés ont fourni l'information nécessaire au calcul de l'Indice d'Evolution (IE) des types d'occupation du sol. L'IE constitue un indice simple pour la mesure des changements du paysage dans le temps. Il s'agit de la proportion totale (\%) de terrain gagné ou perdu pour chaque type d'occupation du sol entre les années 1995 et 2010. Cette proportion est le résultat de la relation entre les pertes de surface en faveur des autres types d'occupation du sol et les surfaces gagnées durant les deux périodes concernées. Pour apprécier l'évolution des différentes unités d'état de surface de 1995 à 2010, les opérations suivantes ont été réalisées :

- U U995, la superficie d'une unité d'état de surface en 1995 ;

- $\quad U_{2010}$, la superficie de la même unité d'état de surface en 2010 ;

- $\quad \mathrm{U}_{1}$, la variation de la superficie de ladite unité d'état de surface de 1995 à 2010.

$\mathrm{U} 1=\mathrm{U}_{2010}-\mathrm{U}_{1995} ;$ ainsi si :

- $\quad \mathrm{U}_{1}=0$, alors il y a stabilité ;

- $\mathrm{U}_{1}>0$, alors il y a évolution progressive ;

- $\quad \mathrm{U}_{1}<0$, alors il y a une évolution régressive.

Ces différentes analyses ont permis d'obtenir les résultats qui permettent d'apprécier l'évolution spatio-temporelle du paysage urbain et agraire dans la Commune de Ouidah ainsi que leurs déterminants.

\section{Résultats}

\subsection{Modes d'accès à la terre en évolution}

Les modes courants d'accès à la terre dans la commune demeurent la succession ou l'héritage, la location, l'achat et le don (figure 3). Les exploitations familiales sont généralement pratiquées sur les terres héritées. Les superficies cultivées sont en moyenne de $800 \mathrm{~m}^{2}$. Le nombre de champs par paysan est de 1 à 3 . 


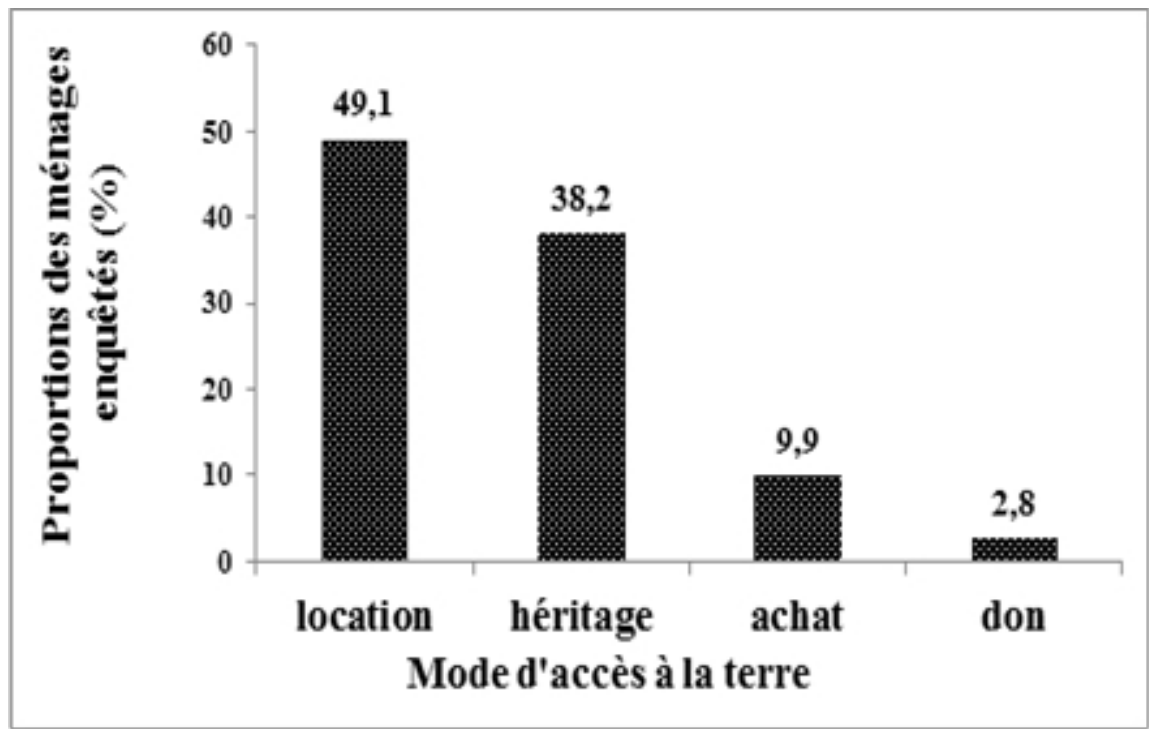

Figure 3. Modes d'accès à la terre dans la Commune de Ouidah

L'analyse de la figure 3 montre que la location (49\%) reste le mode d'accès à la terre le plus dominant suivi de l'héritage (38,2\%). La location est plus courante à Savi que dans les autres arrondissements. Dans la Commune de Ouidah, tous les enfants quel que soit leur sexe sont bénéficiaires de l'héritage foncier (78,3\% des répondants). Le partage de l'héritage se fait soit en fonction de l'âge, soit à part égale au niveau des enfants. Il existe une différence entre les modes de partage au niveau des arrondissements. A Gakpé par exemple, le partage est plus en fonction de l'âge ( $82,7 \%$ des répondants). A Pahou par contre, le partage se fait à part égale $(98 \%)$ entre les héritiers. Ceux qui n'ont bénéficié que d'une superficie très réduite sont amenés à vendre leur part. Or, selon les enquêtés ( $89 \%$ ), dans la Commune de Ouidah, la terre est sacrée. De ce fait, sa vente n'intervenait auparavant, qu'en cas de force majeure (maladie, décès, inscription à l'université, construction de maison). Aujourd'hui, la vente de la terre pour des finalités d'achats de moto, d'organisation des cérémonies funéraires ou religieuses et de mariage a pris le dessus sur d'autres considérations. De toutes les façons, les modes de partage accentuent l'émiettement des champs.

Dans le secteur d'étude, trois (03) catégories d'acquéreurs se distinguent: les petits acquéreurs, les acquéreurs moyens et les grands acquéreurs. Les petits acquéreurs sont ceux qui achètent des parcelles dont les dimensions varient entre 0 à 1 ha et les acquéreurs moyens sont ceux qui achètent des terrains dont les dimensions varient entre 1 à 5 ha. Quant aux grands acquéreurs, ce sont ceux qui achètent des domaines dont les dimensions sont supérieures à 5 ha. Les terres achetées sont utilisées pour la plantation des ligneux. Le prix de la terre varie d'un arrondissement à un autre, d'un village 
à un autre et tient compte des facteurs tels que le caractère inondable de la zone en période de crue, de la présence d'infrastructures sociocommunautaires, de la présence ou non des marécages ou de bas-fonds. L'acquéreur peut payer au comptant ou par tranche. Lorsque le prix de la cession de la parcelle est payé, l'acquéreur rentre en possession de sa convention de vente. Le prix de la parcelle $\left(400 \mathrm{~m}^{2}\right)$ varie entre 500000 à 2 000000 de francs CFA selon les arrondissements. Les nouveaux acquéreurs sont des fonctionnaires, des commerçants et de plus en plus des ouvriers et des paysans. La location existe sous sa forme la plus courte. Elle dure une année civile et demie et permet aux producteurs d'exploiter la terre pendant deux grandes saisons de pluie et une petite saison de pluie. La location se fait par "kanti" (parcelle de $400 \mathrm{~m}^{2}$ ), son prix varie entre 1500 à 2000 francs CFA et le locataire n'est pas autorisé à mettre les cultures pérennes.

Les agriculteurs périurbains de la Commune cultivent, pour la plupart, sur des terrains dont ils n'ont toujours pas la maîtrise foncière. L'accès au foncier est la contrainte majeure dans toute la Commune et l'une des sources de différenciation des types de culture, des systèmes de production et des revenus car le problème foncier revêt deux aspects fondamentaux : d'une part les espaces bâtis se développent réduisant ainsi les terres cultivables et d'autre part, il y a un mode d'accès (location) qui ne favorise pas une bonne mise en valeur. Les producteurs emblavent différentes superficies de terres cultivables pour leurs activités agricoles. Elles sont subdivisées en trois classes bien distinctes : la petite classe $\left(0\right.$ à $\left.1000 \mathrm{~m}^{2}\right)$, la moyenne classe ( 1 à $\left.2 \mathrm{ha}\right)$ et la grande classe (plus de 2 ha). En effet, 55,66 \% des enquêtés exercent leur activité sur de petites parcelles, 37,74\% sur des parcelles à dimension moyenne et $6,60 \%$ exploitent de grandes superficies cultivables. Dans ce cas, les outils utilisés ne peuvent être que traditionnels. La pratique de jachère est rare et lorsqu'elle est pratiquée, elle est de courte durée ( 1 an).

\subsection{Propension du paysage périurbain associée à la déprise agricole}

Les figures 4 et 5 présentent les cartes d'occupation du sol obtenues après digitalisation des photographies aériennes de 1995 et 2010. Elles montrent la distribution spatiale des sept unités d'occupation du sol identifiées ainsi que la proportion du paysage occupée par les différentes catégories et les relations entre leur surface totale. 


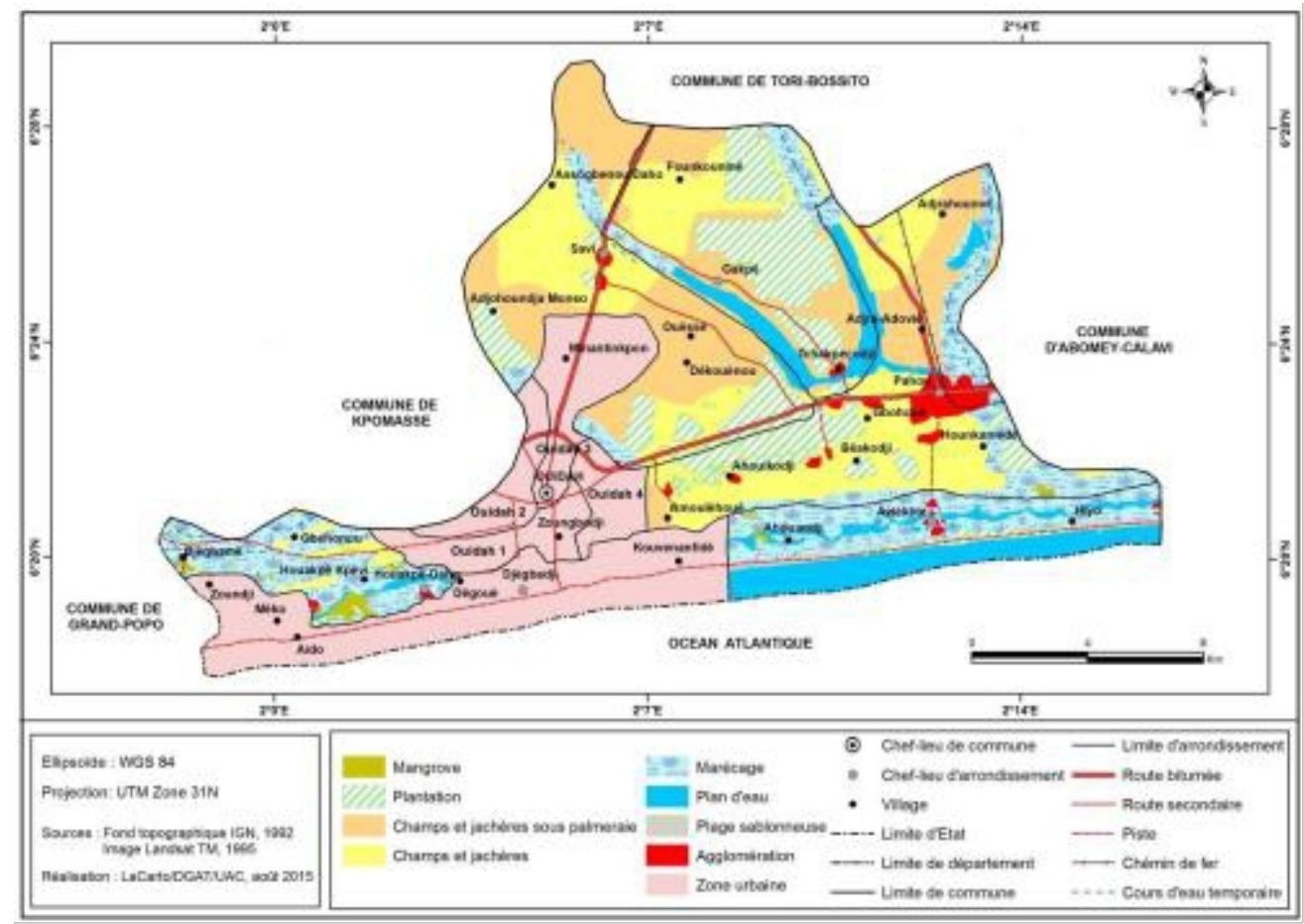

Figure 4 : Occupation du sol de la zone périurbaine en 1995

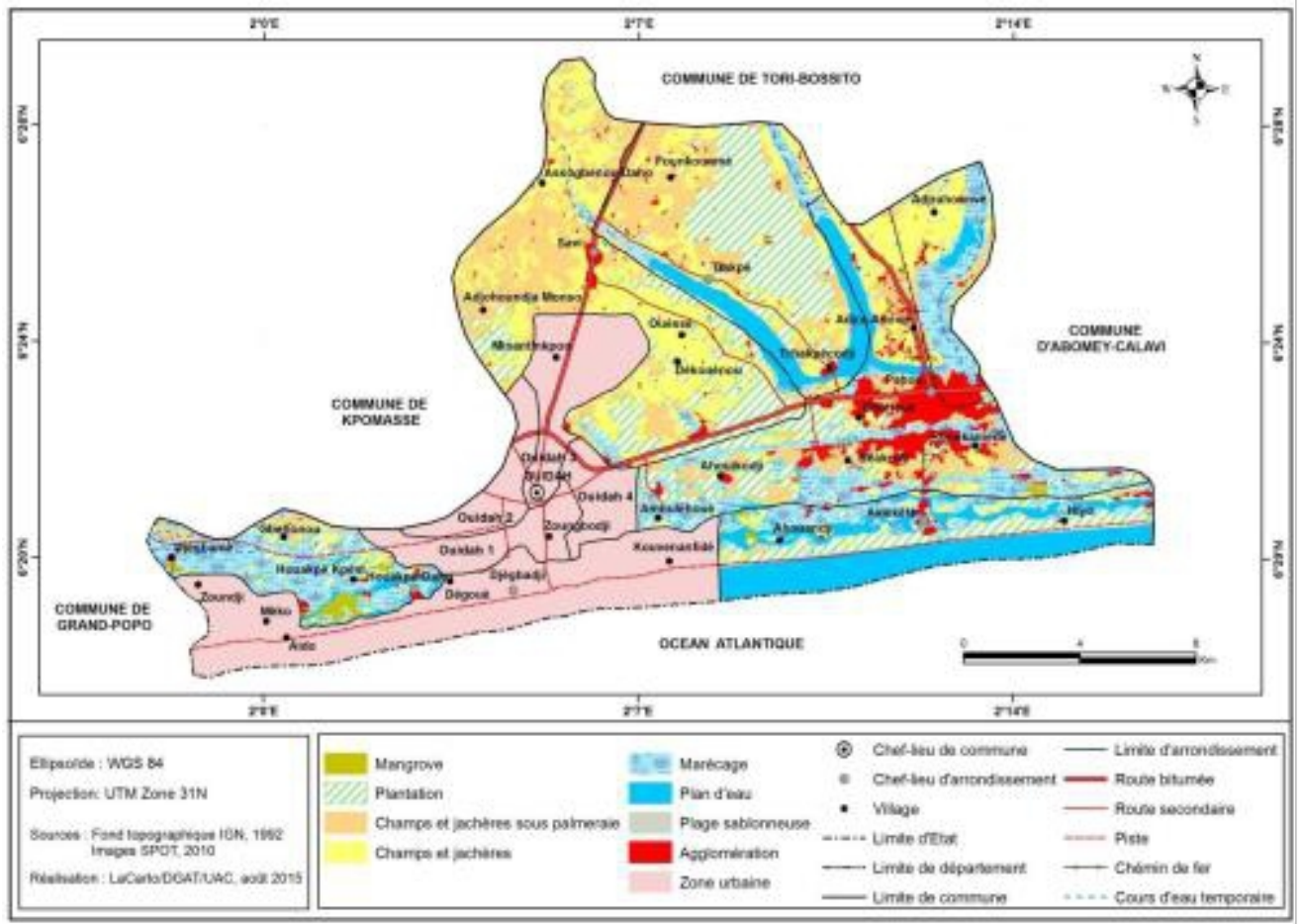

Figure 5 : Occupation du sol de la zone périurbaine en 2010 
L'analyse de l'occupation du sol de 1995 et de 2010 a permis d'apprécier la dynamique de l'occupation du sol (tableau 2).

Tableau 2 : Proportions des unités d'occupation du sol en 1995 et 2010

\begin{tabular}{lcccc}
\hline \multirow{2}{*}{ Unités d'occupation du sol } & \multicolumn{2}{c}{1995} & \multicolumn{2}{c}{2010} \\
\cline { 2 - 5 } & $\begin{array}{c}\text { Superficie } \\
\left(\mathrm{km}^{2}\right)\end{array}$ & $\begin{array}{c}\text { Pourcentage } \\
(\%)\end{array}$ & $\begin{array}{c}\text { Superficie } \\
\left(\mathrm{km}^{2}\right)\end{array}$ & $\begin{array}{c}\text { Pourcentage } \\
(\%)\end{array}$ \\
\hline Mangrove & 1,97 & 0,79 & 1,67 & 0,67 \\
Plantation & 39,41 & 15,83 & 57,40 & 23,05 \\
Champs et jachères sous palmeraie & 55,60 & 22,33 & 46,34 & 18,61 \\
Champs et jachères & 62,70 & 25,18 & 52,26 & 20,99 \\
Marécage & 52,32 & 21,01 & 45,90 & 18,43 \\
Plan d'eau & 28,82 & 11,57 & 28,78 & 11,56 \\
Plage sablonneuse & 2,09 & 0,84 & 1,52 & 0,61 \\
Agglomération & 6,10 & 2,45 & 15,14 & 6,08 \\
\hline Total & 249 & 100 & 249 & 100 \\
\hline
\end{tabular}

Source : Résultats d'interprétation des cartes, 2018

En 1995, le paysage apparaît plus rural, dominé par les plantations $(39,41 \%)$, les champs et jachères $(25,18 \%)$, les champs et jachère sous palmeraie $(22,33 \%)$ et les marécages $(21,01 \%)$. L'analyse de la relation surface unité /surface totale (tableau 2) révèle que les espaces cultivés occupent une surface de $212 \mathrm{~km}^{2}$ alors que les autres unités (mangrove, plage sablonneuse, agglomération, plan d'eau) ne couvrent qu'une superficie de 37 $\mathrm{km}^{2}$. Ces chiffres illustrent la prédominance des activités agricoles à ce moment. D'une manière générale, les plantations, les champs et jachères sous palmeraie et les champs et jachères se distribuent de façon concentrée et compacte. Elles constituent un ensemble d'unités d'occupation du sol le long des plans d'eau et marécages interrompues par des habitats et des chemins. Cependant, il y a des secteurs où les marécages sont interrompus par la mangrove. Par ailleurs, les marécages sont présents dans tout le secteur d'étude, notamment à Avlékété et à Houakpè où ils constituent l'essentiel de l'unité d'occupation du sol à l'intérieur de laquelle sont identifiés quelques îlots d'agglomérations.

En 2010, le paysage a subi certaines transformations, représentées principalement par le recul et la fragmentation de l'espace agricole, le recul de la mangrove, des plans d'eau et de la plage sablonneuse (figure 5). L'expansion de la plantation et le développement de la sylviculture. La surface des cultures a décru de $212 \mathrm{~km}^{2}$ à $144,50 \mathrm{~km}^{2}$. Dans le cas de la mangrove, des plans d'eau et de la plage sablonneuse, on assiste respectivement à une diminution de leurs surfaces de 1,97 à $1,67 \mathrm{~km}^{2}$, de 28,82 à $28,78 \mathrm{~km}^{2}$ et de 2,09 à $1,52 \mathrm{~km}^{2}$. Les champs et les jachères sous palmeraie, les champs et les jachères et l'agglomération apparaissent spatialement plus fragmentés et mélangés avec les autres types d'occupation du sol. 
L'évolution des types d'occupation du sol indique la dynamique du paysage. Puisque la structure paysagère n'est pas stable, les changements du paysage durant les 15 années ont été reconnus à travers l'identification des transitions et des tendances évolutives des types d'occupation du sol entre les années 1995 et 2010. Ces mutations du paysage sont quantifiées, puis évaluées ensuite à travers la matrice de transition et l'Indice d'Evolution (tableau 3).

Tableau 3 : Matrice de transition des unités d'occupation du sol entre 1995 et 2010

\begin{tabular}{|c|c|c|c|c|c|c|c|c|c|}
\hline \multirow{2}{*}{$\begin{array}{c}\text { Unités de } \\
1995\end{array}$} & \multicolumn{8}{|c|}{ Unités de 2010} & \multirow{2}{*}{$\begin{array}{c}\text { Superficie } \\
\text { totale en } \\
2010\left(\mathrm{~km}^{2}\right)\end{array}$} \\
\hline & MG & PL & CJP & CJ & FM & $\mathbf{P E}$ & PS & AG & \\
\hline MG & 1,66 & 0 & 0 & 0 & 0,18 & 0,13 & 0 & 0 & 1,97 \\
\hline PL & 0 & 25,24 & 6,11 & 5,10 & 2,13 & 0 & 0,23 & 0,60 & 39,41 \\
\hline CJP & 0 & 10,49 & 14,76 & 24,51 & 4,20 & 0 & 0 & 1,65 & 55,60 \\
\hline CJ & 0 & 10,16 & 21,11 & 16,69 & 9,34 & 0 & 0 & 5,39 & 62,70 \\
\hline FM & 0,01 & 10,50 & 4,35 & 5,92 & 29,95 & 0 & 0 & 1,58 & 52,32 \\
\hline PE & 0 & 0 & 0 & 0 & 0 & 27,89 & 0,93 & 0 & 28,82 \\
\hline PS & 0 & 1,00 & 0,01 & 0,04 & 0,10 & 0,75 & 0,16 & 0,03 & 2,09 \\
\hline $\mathbf{A G}$ & 0 & 0 & 0 & 0 & 0 & 0 & 0,20 & 5,89 & 6,1 \\
\hline $\begin{array}{c}\text { Superficie } \\
\text { totale en } \\
2010\left(\mathbf{k m}^{2}\right)\end{array}$ & 1,67 & 57,40 & 46,34 & 52,26 & 45,90 & 28,78 & 1,52 & 15,14 & 249 \\
\hline
\end{tabular}

Source : Image Spot 1995 et LandSat ETM+2010

Légende : MG : Mangrove; CJP : Champs et jachères sous palmeraie; CJ : Champs et jachères ; FM : Formation marécageuse ; PE : Plan d'eau ; PS : Plage sablonneuse ; AG : Agglomération ; PL : Plantation

Dans les cellules des lignes, se trouvent respectivement les formations végétales et les autres unités d'occupation du sol de 1995 et de 2010. Les conversions se font des lignes vers les colonnes. Les cellules de la diagonale correspondent aux unités qui sont demeurées stables de 1995 à 2010. Les unités qui sont en dehors de la diagonale représentent les changements de végétation et d'autres unités d'occupation du sol.

L'examen de la matrice permet de retenir 8 classes d'occupation du sol aussi bien en 1995 qu'en 2010. De 1995 à 2010, la superficie de la mangrove forêts galeries est passée de $1,97 \mathrm{~km}^{2}$ à $1,67 \mathrm{~km}^{2}$ soit une diminution de 15,22 $\%$. La matrice de transition montre que $0,18 \mathrm{~km}^{2}$ de la mangrove est convertie en formation marécageuse et $0,13 \mathrm{~km}^{2}$ de cette espèce végétale en plan d'eau. Ces différentes valeurs prouvent que la mangrove connait une évolution régressive de 1995 à 2010.

S'agissant de l'évolution des plantations, elles ont connu une augmentation entre 1995 et 2010 avec un taux d'évolution global de 45,64 $\%$. En effet, leur superficie est passée de $39,41 \mathrm{~km}^{2}$ en 1995 à $57,40 \mathrm{~km}^{2}$ en 2010. La matrice de transition révèle que $10,49 \mathrm{~km}^{2}, 10,16 \mathrm{~km}^{2}, 10,50 \mathrm{~km}^{2}$, $1,00 \mathrm{~km}^{2}$ représentant respectivement, les superficies des champs et jachères 
sous palmeraie, les champs et jachère, de la formation marécageuse et de la plage sablonneuse se sont converties en plantations.

Concernant l'évolution des champs et jachères sous palmeraie, les savanes arbustives et arborées ont connu une régression entre 1995 et 2010 avec un taux de régression global de 16,65\%. En effet, leur superficie est passée de 55,60 $\mathrm{km}^{2}$ en 1995 à 46,34 $\mathrm{km}^{2}$ en 2010. La matrice de transition révèle que $24,51 \mathrm{~km}^{2}, 4,20 \mathrm{~km}^{2}$ et $1,64 \mathrm{~km}^{2}$ des champs et jachères sous palmeraie se sont convertis respectivement, en champs et jachères, en formation marécageuse et en agglomération.

Entre 1995 et 2010, la superficie des champs et jachères a été converti en formations marécageuses $\left(9,34 \mathrm{~km}^{2}\right)$ et agglomération $\left(5,39 \mathrm{~km}^{2}\right)$, soit un taux de régression de $16,49 \%$. La superficie des formations marécageuses est passée de $52,32 \mathrm{~km}^{2}$ à $45,90 \mathrm{~km}^{2}$ soit une régression de $12,27 \%$ au profit des agglomérations. A la même période, la superficie des plans d'eau est passée de $28,82 \mathrm{~km}^{2}$ à $28,78 \mathrm{~km}^{2}$, soit une régression de $0,24 \%$ au profit de la plage sablonneuse. La superficie de la plage sablonneuse est passée de 2,09 $\mathrm{km}^{2}$ à $1,52 \mathrm{~km}^{2}$, soit une régression de 27,27 \% au profit de l'agglomération de 1995 à 2010 .

Quant aux agglomérations, entre 1995 et 2010, elles sont passées de $6,1 \mathrm{~km}^{2}$ à $15,14 \mathrm{~km}^{2}$ soit une augmentation de $148,19 \%$ au détriment des plantations, des champs et jachères sous palmeraie, des champs et jachères, de la formation marécageuse et de la plage sablonneuse.

L'Indice d'Evolution (IE) permet de mieux apprécier les types d'occupation du sol dans le secteur d'étude (tableau 4).

Tableau 4 : Indice d'Evolution (IE) des types d'occupation du sol entre 1995 et 2010

\begin{tabular}{lcccc}
\hline Unités d'occupation du sol & $\begin{array}{c}\text { Superficie } \\
\left(\mathrm{km}^{2}\right) \\
\text { en } 1995\end{array}$ & $\begin{array}{c}\text { Superficie } \\
\left(\mathrm{km}^{2}\right) \\
\text { en } 2010\end{array}$ & $\begin{array}{c}\text { DifférenceSu } \\
\text { perficie }\left(\mathrm{km}^{2}\right)\end{array}$ & $\begin{array}{c}\text { Indice } \\
\text { d'évolution (\%) }\end{array}$ \\
\hline Mangrove & 1,97 & 1,67 & $-0,30$ & $-0,12$ \\
Plantation & 39,41 & 57,40 & $+17,79$ & $+7,22$ \\
Champs et jachères sous palmeraie & 55,60 & 46,34 & $-9,09$ & $-3,72$ \\
Champs et jachères & 62,70 & 52,26 & $-10,44$ & $-4,19$ \\
Marécage & 52,32 & 45,90 & $-6,42$ & $-2,58$ \\
Plan d'eau & 28,82 & 28,78 & $-0,16$ & $-0,02$ \\
Plage sablonneuse & 2,09 & 1,52 & $-0,57$ & $-0,23$ \\
Agglomération & 6,10 & 15,14 & $+9,04$ & $+3,63$ \\
\hline Total & 249 & 249 & - & - \\
\hline
\end{tabular}

Source : Image Spot 1995 et LandSat ETM+2010

La période allant de 1995 à 2010 est marquée par le recul de la mangrove $(\mathrm{IE}=-0,12)$, des champs et jachères sous palmeraie $(\mathrm{IE}=-3,72)$, des champs et jachères $(\mathrm{IE}=-4,19)$; des marécages $(\mathrm{IE}=-2,5)$, des plans d'eau $(\mathrm{IE}=-0,02)$; des plages sablonneuses ( $\mathrm{IE}=-0,02)$. Par contre, les plantations et les agglomérations connaissent une augmentation avec les 
indices d'évolution respectivement égale à $+7,22$ et $+3,63$. La réduction des surfaces des autres unités d'occupation est une conséquence du développement des agglomérations et des plantations. En effet, une proportion de $12,91 \%$ du paysage s'est transformée en plantations. Les agglomérations se sont vues aussi accrues par la récupération de terrains $(3,71 \%)$ des plantations, des champs et jachères sous palmeraie, des champs et jachères, des formations marécageuses et de la plage sablonneuse.

Entre 1995 et 2010, les agglomérations $(\mathrm{IE}=+3,63)$ et les plantations (IE $=+7,22)$ se sont étendues considérablement. Au total, les surfaces en agglomérations et en plantations auraient gagné $16,62 \%$ du paysage en conversion au cours de la période considérée. Cette situation a permis à la Commune de Ouidah en 2010, d'être occupée à 29,13\% par ces agglomérations et plantations. En terme clair, les tendances évolutives peuvent être regroupées en cinq (05) types principaux de mutations : les transformations de l'espace agricole, la dégradation de l'espace agricole, la déforestation, le déboisement, l'urbanisation.

Les transformations de l'espace agricole sont liées à des changements internes de la structure agraire. La période allant de 1995 à 2010 comprend une déstructuration des anciennes activités rurales (champs et jachères sous palmeraie, champs et jachères) représentée par la mutation en plantation et en formations marécageuses et vice-versa. Le secteur rural de 1995 à 2010 a subi une restructuration. Cette dernière est représentée par l'extension des plantations d'arbres. Pendant plusieurs années, des carrières ouvertes sur la plage sablonneuse de Ouidah sont exploitées. Des centaines de mètres cube de sable y ont été prélevées. Ainsi, la plage sablonneuse s'est dégradée par endroits en des formations marécageuses et en des plans d'eau. Les palétuviers, principales formations forestières (mangrove) sont coupés et utilisés comme bois de chauffe. La mangrove est ainsi détruite au profit de quelques formations marécageuses. De 1995 à 2010, plusieurs espaces occupés par des plantations sont mis à nu à des fins agricoles (champs et jachères sous palmeraie ou champs et jachères) ou bien pour recevoir des habitations (extension de l'agglomération).

Il ressort que les surfaces en agglomérations et en plantations ont gagné 16,62 \% du paysage en conversion entre 1995 et 2010. Cet état de chose concède à la Commune de Ouidah une occupation de $29,13 \%$ en 2010. Des espaces périurbains, autrefois utilisés à des fins agricoles (champs et jachères sous palmeraie ou champs et jachères), sont aujourd'hui occupés par des habitations. Le mouvement d'urbanisation dans la Commune de Ouidah prend de l'ampleur d'année en année avec ses corollaires. Ainsi, les superficies cultivables s'amenuisent. L'accès à la terre constitue désormais un casse-tête pour les producteurs agricoles périurbains et la location est le mode dominant. 
En conséquence, $66 \%$ des exploitants emblavent des superficies comprises entre 0 et 1 ha; donc de petits espaces.

\section{Discussion}

Les modes d'accès à la terre courants dans la localité de Ouidah demeurent la succession ou l'héritage, la location, l'achat et le don. Ces principaux modes d'accès à la terre sont enregistrés à Parakou et dans la Commune de Tchaourou par I. Yolou (2015, p. 138) et I. Yolou et al. (2018, p. 573). Ils sont confirmés par A. Y. Tohozin et al. (2012, p. 5) au cours de leur étude sur la gestion du foncier et la dynamique des espaces agricoles dans la Commune de Tori-Bossito et par B. Fangnon (2012, p.201), T. R. G. Kadjegbin et al. (2018, p.417) et I. Yolou et al. (2019, p. 79) qui ont également recensé les mêmes modes d'accès à la terre respectivement dans le département du Couffo et dans les Communes de Dassa-Zoumè et de Glazoué et de Covè. A Ouidah, tous les enfants quel que soit leur sexe sont bénéficiaires de l'héritage foncier (78,3\% des répondants). Le partage de l'héritage se fait soit en fonction de l'âge, soit à part égale au niveau des enfants. L'héritage foncier est un transfert de génération en génération des terres et leurs ressources (I. Yolou et al., 2019, p. 79). Selon I. Yolou et al. (2018, p. 573), l'effet combiné de la croissance démographique et des règles d'héritage entraînent le morcellement des terres et restent la cause la plus actuelle du changement du droit foncier coutumier vers des droits plus individualisés. De plus, à cause des modes de transmission par héritage, la taille des champs se réduira de plus en plus; d'où les conflits fonciers (A. Y. Tohozin et al., 2012, p. 6). En moyenne, chaque ménage dispose de 2 champs couvrant une superficie moyenne de 1,6 ha. Près de 96,8\% des champs ont une taille inférieure à 3 ha, et environ $1 \%$ ont une taille de plus de 5 ha. Les champs sont donc de taille très petite pour favoriser une mise en valeur conséquente. La location est plus courante à Savi que dans les autres arrondissements de la Commune de Ouidah. Cette situation se justifie par le fait que ce sont les fils adultes qui sont héritiers des terres dans la mesure où, après la mort de leur père, ils se chargent de l'éducation de leurs jeunes frères. Ce faire-valoir constitue un véritable handicap aux producteurs à cause de l'insécurité foncière (op. cit).

Des espaces, autrefois utilisés à des fins agricoles (champs et jachères sous palmeraie ou champs et jachères), sont aujourd'hui péri-urbanisés et donc occupés par des habitations et autres établissements humains. Le mouvement d'urbanisation dans la Commune de Ouidah prend de l'ampleur d'année en année. En effet, les travaux de S. Paquette et G. Domon (1999) cités par J. Ruiz (2009, p. 21) confirment cet état de fait en montrant comment les dynamiques agricoles s'étaient peu à peu dissociées des dynamiques démographiques au point où des municipalités marquées par le déclin de l'agriculture sont le siège d'une croissance démographique sans précédent 
avec la venue de nouvelles populations d'origine urbaine. Ce qui fait qu'aujourd'hui, l'on distingue deux grands types d'espaces aux tendances agricoles divergentes : les zones d'agriculture intensive et les zones de déprise agricole. Par ailleurs, selon M. Kanda et al. (2017, p. 1), l'urbanisation et la légitimation de la place de l'agriculture dans le plan urbain ainsi que son intégration dans la gestion du paysage et de l'espace conditionnent la pérennité de cette agriculture. Car, l'urbanisation à proximité des centres urbains est un second phénomène qui a conduit à la perte de superficies de terres agricoles (J. Ruiz, 2009, p. 49). L'étalement des villes engendre une compétition foncière qui ne laisse guère d'espaces pour les activités agricoles (M. Kanda et $a l ., 2017$, p. 9). Cette croissance urbaine se traduit par une forte demande en terres à bâtir et est largement alimentée par le développement des maisons individuelles. Par contre, le rapport du PNUD (2015, p. 5) montre plutôt que le nombre élevé de petits exploitants et la faiblesse de la législation foncière induisent un morcellement de plus en plus accentué des terres avec pour conséquence, la disparition progressive des terres de production agricole et des aires de pâturages. Cependant, M. T. Demaze (2008, p. 2) insiste en montrant que l'augmentation continue de la population entraîne une pression foncière et un accroissement des besoins en terre d'autant plus importants que la grande majorité du territoire. Cette gestion de la terre a dès lors fait émerger des enjeux de l'expansion urbaine dans les banlieues des grandes villes du Bénin (K. D. Goungni et al., 2016, p.118). Selon B. Baccaïni et F. Semecurbe (2009, p. 3), l'étalement urbain s'explique alors, pour l'essentiel, par un « débordement » de la ville, du fait d'arrivées plus nombreuses que les départs, dans les villes en forte croissance démographique. Une partie des zones agricoles ou naturelles des périphéries urbaines se transforme en zones dites « artificielles », principalement sous l'effet de la construction de logements individuels. En fait, pour les pays en développement, il ne s'agit donc plus de penser le modèle agricole uniquement sur la productivité, mais bien de le repenser en intégrant des préoccupations de durabilité, de résilience et d'accessibilité aux petits producteurs (G. Bayiha, 2016, p.4). C'est pourquoi, selon A. Y. Tohozin et al. (2012, p. 11), une réforme agraire s'impose pour réguler la gestion foncière. En effet, une bonne législation relative au foncier rural et urbain s'impose pour limiter les risques de l'aggravation de la précarité des ménages agricoles et les protéger contre l'assaut des courtiers et autres commerçants véreux obnubilés par l'acquisition de vastes domaines.

\section{Conclusion}

Ce travail a permis d'apporter une contribution à la réflexion sur la question de l'urbanisation et ses effets de déprise agricole dans la Commune de Ouidah. Le grignotage des espaces ruraux s'intensifie et le phénomène de périurbanisation se poursuit considérablement dans ce milieu d'étude. Cette 
pression se traduit par l'émiettement des espaces agricoles et l'acquisition des terres par les citadins. Dans les localités rurales plus ou moins proches de l'espace urbain, la terre est sujette à une demande accrue et fait l'objet d'intenses transactions à travers des systèmes d'achat et de vente et des processus d'accaparement individualisés. En conséquence, la rareté ou la pénurie des terres autour de la ville historique de Ouidah, oblige certains habitants à s'installer dans les localités environnantes à la recherche de terres fertiles ou à abandonner carrément les activités agricoles. Ces résultats font appel à des actions concertées pour assurer une cohabitation équilibrée des espaces urbanisés avec les surfaces agricoles, gage d'un développement local durable de la Commune de Ouidah. L'élaboration des documents de planification territoriale : Plan de Développement Communal (PDC), Schéma Directeur d'Aménagement de la Commune (SDAC), etc. devra tenir compte de cet impératif.

\section{References :}

1. ALLEN Thomas, HEINRIGS Philipp et ZOUNDI Sibiri Jean, 2015, Mutations de l'économie agro-alimentaire et implications, rapport ECOWAP+10, 24 p.

2. BACCAÏNI Brigitte et SEMECURBE François, 2009, La croissance périurbaine depuis 45 ans : Extension et densification, Pôle Analyse territoriale, Insee, $\mathrm{N}^{\circ} 1240,4 \mathrm{p}$.

3. BAYIHA Gerard, MATHE Syndhia, TEMPLE Ludovic, 2016, Diversité des trajectoires vers l'agriculture biologique dans les pays en développement: Le cas du Cameroun. 10ème journées de recherche en sciences sociales (IESIEG),, Paris, France. 25 p.

4. CASADO Ana, 2007, Etude de la structure et de la dynamique des paysages de montagne, Master 2 Géoenvironnement. Département de Géographie à Université Blaise Pascal, Laboratoire de géographie physique UMR 6042- CNRS, 51 p.

5. DEMAZE Moise Tsayem, 2008, «Croissance démographique, pression foncière et insertion territoriale par les abattis en Guyane française », Norois, Presses universitaires de Rennes, pp.111-127.

6. FANGNON Bernard, 2012, Qualité des sols, systèmes de production agricole et impacts environnementaux et socio-économiques dans le Département du Couffo au sud-ouest du Bénin, Thèse de Doctorat de Géographie, EDP/FLASH/UAC, 308 p.

7. GLELE Afiavi Gisèle, 2015, La périurbanisation et les dynamiques foncières sur le plateau d'Allada (Sud-Bénin) : l'espace témoin de la Commune d'Abomey-calavi, Thèse unique de doctorat, Université d'Abomey-Calavi, $451 \mathrm{p}$.

8. GOUNGNI Kouassi Darius, AKIYO Offin Lié Rufin, AFFO Fabien, 
Albert NOUHOUAYI et N'BESSA Benoit, 2016, « Les enjeux socioéconomiques de l'expansion urbaine dans les banlieues des grandes villes au Bénin : exemple de Hêvié », Revue Mu Kara Sani de l'Institut de Recherches en Sciences Humaines (IRSH) de l'Université ABDOU MOUMOUNI de Niamey (Niger), Vol. 24, pp. 106-123

9. INSAE (Institut National de la Statistique et de l'Analyse Economique), 2018, Evolution de la population du Bénin, Rapport, 27 $\mathrm{p}$.

10. KADJEGBIN Toundé Roméo Gislain, YABI Ibouraima, ADJAKPA Tchékpo Théodore, KOTCHARE Parfaite, SEWADE Sokegbe Grégoire, HOUSSOU Christophe Sègbè, 2018, «Influences des modes d'accès à la terre sur la production agricole dans les Communes de Dassa-Zoumé et de Glazoué au centre du Bénin », European Scientific Journal. Vol.14, No.6. p. 412-431.

11. KANDA Madjouma, BADJANA Hèou Maléki, FOLEGA Fousseni, AKPAVI Sêmihinva, WALA Kpérkouma, IMBERNON Jacques et AKPAGANA Koffi, 2017, «Dynamique centrifuge du maraîchage périurbain de Lomé (Togo) en réponse à la pression foncière », Cahiers Agricultures 2017, 26 : 15001, pp.1-10

12. PAQUETTE Sylvain et DOMON Gérard, 2000, « Le paysage comme agent de recomposition des communautés rurales du sud du Québec : Nouvelles possibilités, nouvelles exigences ». In: M. Carrier et S. Côté, (dir.). Gouvernance et territoires ruraux. Éléments d'un débat sur la responsabilité du développement. Sainte-Foy, Presses de l'Université du Québec. pp. 190-222.

13. PNUD, 2015, Agriculture, sécurité alimentaire et développement humain au Bénin, Rapport national sur le développement humain, 12 p.

14. RUIZ Julie et DOMON Gérald, 2005, « Les paysages de l'agriculture en mutation ». In : Poullaouec-Gonidec, P., Domon, G. et S. Paquette (Éds.). Paysages en perspective. Presses de l'université de Montréal, série « Paysages », Montréal, pp. 47-97

15. RUIZ Julie, 2009, Réintroduire la multifonctionnalité des paysages en zone d'intensification agricole : contribution des approches intégrées en écologie du paysage, Thèse de doctorat, Faculté de l'aménagement, Université de Montréal, 237 p.

16. TOHOZIN Antoine Yves, GLELE Gisèle Afiavi, FANGNON Bernard et ADJALLALA Odilon, 2012, «Gestion du foncier et dynamique des espaces agricoles au sud du Bénin : cas de la Commune de ToriBossito », J. Rech. Sci. Univ. Lomé (Togo), 2012, Série B, 14(2) : 1 12 
17. YOLOU Isidore, 2015, Agriculture urbaine à Parakou : Etat des lieux et perspectives d'amélioration de l'utilisation des déchets organiques, Thèse de doctorat unique. FLASH/UAC, $330 \mathrm{p}$.

18. YOLOU Isidore, DJOBO Kondé, YABI Ibouraima, YABI Afouda Jacob et AFOUDA Fulgence, 2018, « Problématique de la sécurisation des terres et ses effets sur la durabilité des activités agricoles dans la Commune de Tchaourou (centre du Bénin) », Mélanges en hommages aux Professeurs HOUSSOU Christophe Sègbè, HOUNDAGBA Cossi Jean et THOMAS Omer. Volume 2. p. 564-576.

19. YOLOU Isidore, YABI Ibouraima, DJOBO Kondé, DOSSOU Faustin Cakpo, YABI Jacob Afouda, AFOUDA Fulgence, 2019, «Agroforesterie à base de Elaeis guineensis en tant qu'option du développement durable dans la Commune de Covè (sud-est du Bénin) : possibilités, pratiques et limites », Revue Ivoirienne de Géographie des Savanes, Numéro 6, pp. 66-89. 